\title{
Impact of rubella vaccination strategy on the occurrence of congenital rubella syndrome
}

\author{
Impacto da vacinação contra rubéola na ocorrência da síndrome \\ da rubéola congênita \\ Tatiana Miranda Lanzieri' ${ }^{1}$, Diana Pinto ${ }^{2}$, D. Rebecca Prevots ${ }^{3}$
}

\begin{abstract}
Resumo
Objetivo: A vacinação rotineira contra a rubéola foi implementada na Paraíba, nordeste brasileiro, através de uma campanha de catch-up direcionada a crianças com idades entre $1 \mathrm{e}$ 11 anos em 1998. Uma campanha entre as mulheres em idade reprodutiva foi realizada em 2001. Descrevemos a epidemiologia da rubéola e da síndrome da rubéola congênita (SRC) no estado da Paraíba entre 1999 e 2005.
\end{abstract}

Métodos: Foram analisados os dados de vigilância da rubéola e da SRC entre 1999 e 2005. Os casos suspeitos de rubéola foram confirmados por exames laboratoriais, vínculo epidemiológico ou por critério clínico. Foram utilizadas as definições internacionais padrão de SRC.

Resultados: Dos 5.924 casos suspeitos de rubéola entre 1999 e $2005,1.266(21 \%)$ foram confirmados, 766 (61\%) deles através dos exames laboratoriais. Durante uma epidemia de rubéola em 2000 $(18,8 / 100.000)$, a incidência entre os indivíduos com idades entre 14 e 19 anos (42/100.000) quadruplicou em relação a crianças com idades entre 1 e 13 anos (intervalo de confiança de $95 \%=3,2-5,1$ ). A incidência geral da rubéola foi de 0,9/100.000 em 2005. Dos 177 casos suspeitos de SRC entre 1999 e 2005, 167 (94\%) foram testados para a presença de IgM. Dos 14 (8\%) casos de SRC confirmados laboratorialmente, 12 (86\%) haviam nascido em 2001, com uma incidência de $0,2 / 100.000$ crianças menores de 1 ano nesse ano.

Conclusões: A campanha de vacinação contra a rubéola em 1998 não foi suficiente para prevenir um surto entre adultos jovens em 2000, com uma alta incidência de SRC em 2001. Entre 2002 e 2005, as incidências de rubéola e de SRC diminuíram; entretanto, a alta cobertura da vacina e a vigilância de alta qualidade são importantes para que se atinja a erradicação da SRC até 2010.

J Pediatr (Rio J). 2007;83(5):415-421: Rubéola, surtos, síndrome da rubéola congênita, vacinação, Brasil.
Abstract
Objective: Routine rubella vaccination was introduced in Paraíba, northeastern Brazil, through a catch-up campaign targeting children aged 1-11 years, in 1998. A campaign among women of childbearing age was conducted in 2001. We describe the epidemiology of rubella and congenital rubella syndrome (CRS) in the state of Paraíba between 1999 and 2005.

Methods: Rubella and CRS surveillance data for the 1999-2005 period were analyzed. Suspected cases of rubella were confirmed by laboratory findings, epidemiological link, or clinical data. International standardized CRS definitions were used.

Results: Of 5,924 suspected cases of rubella between 1999 and 2005, 1,266 (21\%) were confirmed, $766(61 \%)$ by laboratory findings. During a rubella epidemic in $2000(18.8 / 100,000)$, the incidence among individuals aged $14-19$ years $(42 / 100,000)$ had a fourfold increase relative to children aged $1-13$ years ( $95 \%$ confidence interval $=3 \cdot 2-5 \cdot 1)$. The overall rubella incidence was 0.9/100,000 in 2005. Of 177 suspected cases of CRS between 1999 and 2005, 167 (94\%) were tested for IgM. Of 14 (8\%) laboratory confirmed cases of CRS, 12 (86\%) were born in 2001, with an incidence of $0.2 / 100,000$ children aged less than 1 year.

Conclusions: The 1998 rubella vaccination campaign was insufficient to prevent an outbreak among young adults in 2000, with a high CRS incidence in 2001. Between 2002 and 2005 the incidence of rubella and CRS decreased; however, high routine vaccination coverage and high-quality surveillance remain critically important to achieve CRS elimination by 2010 .

J Pediatr (Rio J). 2007;83(5):415-421: Rubella, outbreaks, congenital rubella syndrome, vaccination, Brazil.

1. MD, MSc. Secretaria de Vigilância em Saúde, Ministério da Saúde, Brasília.

2. MD. Secretaria de Estado de Saúde da Paraíba, João Pessoa, PB.

3. PhD, MPH. National Institute for Allergy and Infectious Diseases, National Institutes of Health, Bethesda, MD, USA.

Apoio financeiro: Ministério da Saúde, Brasil.

Como citar este artigo: Lanzieri TM, Pinto D, Prevots DR. Impact of rubella vaccination strategy on the occurrence of congenital rubella syndrome. J Pediatr (Rio J). 2007;83(5):415-421.

Artigo submetido em 25.05.07, aceito em 06.07.07.

doi 10.2223/JPED.1692 


\section{Introdução}

A rubéola geralmente é uma doença exantemática leve que ocorre na infância, sendo que 25 a $50 \%$ dos casos constituem infecções subclínicas. As conseqüências mais importantes da rubéola resultam da infecção por rubéola no início da gravidez e incluem aborto, aborto espontâneo, natimortalidade e anomalias fetais. Até $80 \%$ dos lactentes concebidos por mães que adquiriram rubéola no primeiro trimestre de gestação podem ser afetados. As anomalias mais comuns associadas à síndrome da rubéola congênita (SRC) são oftalmológicas, cardíacas, auditivas e neurológicas. Embora casos de SRC moderados a graves possam ser reconhecidos prontamente no momento de nascimento, casos leves podem permanecer indetectados durante meses ou até mesmo anos após o nascimento ${ }^{1}$.

O objetivo dos programas de imunização contra rubéola é prevenir a infecção fetal e subseqüente SRC. Muito embora a doença esteja praticamente erradicada em muitos países industrializados, estima-se que 100.000 lactentes nasçam com SRC a cada ano ${ }^{2}$. No Brasil, a vacinação contra a rubéola foi implementada gradativamente pelos estados entre 1992 e $2000^{3}$. A rubéola e a SRC são doenças passíveis de notificação no Brasil desde $1996^{4}$, contudo, a vigilância da rubéola tornou-se mais sensível e representativa somente depois de 1999, quando foi integrada à vigilância do sarampo ${ }^{5}$. Entre 1999 e 2000, grandes surtos de rubéola foram relatados em quase todos os estados brasileiros, com diferentes padrões de distribuição etária entre os estados, provavelmente devido à implementação gradual da vacinação. À medida que casos de rubéola começaram a ser detectados entre gestantes, adotou-se um sistema de vigilância para a SRC em $2000^{4}$ e procedeu-se à vacinação pós-parto. Para acelerar o controle da SRC, realizou-se uma campanha nacional de vacinação, realizada em duas fases, entre 2001 e 2002, direcionada a mulheres em idade reprodutiva ${ }^{6}$. Além disso, em 2003, o Brasil apoiou a resolução da Organização Pan-americana da Saúde que visa erradicar a rubéola e a SRC na região até $2010^{7}$.

No estado da Paraíba, no nordeste brasileiro, a vacinação contra a rubéola foi implementada em agosto de 1998 através de uma campanha de massa utilizando a vacina tríplice viral (sarampo, caxumba e rubéola) em crianças com idades entre 1 e 11 anos, com coberturas entre 49 e $100 \%$ em todos os seus 224 municípios. À campanha se seguiu a implementação da vacina tríplice viral no esquema de vacinação infantil. O esquema atual inclui a primeira dose aos 12 meses e a segunda na idade de 4 a 6 anos. A vigilância da rubéola foi adotada no estado da Paraíba no final de 1998 como parte da intensificação das atividades do programa de eliminação do sarampo. Um grande surto de rubéola com vários casos entre gestantes foi notificado durante o ano 2000. Uma campanha estadual direcionada a mulheres não-grávidas com idades entre 15 e 29 anos foi realizada em novembro de 2001, com coberturas entre 62 e $100 \%$ em todos os municípios. Neste estudo, descrevemos a epidemiologia da rubéola e da SRC no estado da Paraíba entre 1999 e 2005, assim como o impacto da vacinação contra a rubéola.

\section{Métodos \\ Vigilância da rubéola}

A vigilância da rubéola é integrada à do sarampo e inclui: (1) estímulo à vigilância passiva entre profissionais da saúde para a notificação imediata de casos suspeitos de sarampo e rubéola, (2) notificação e investigação imediatas dos casos suspeitos com coleta de sangue para o diagnóstico específico através de testes laboratoriais. Os dados individuais são coletados através de uma ficha de investigação padronizada para sarampo e rubéola, incluindo informações demográficas, manifestações clínicas, histórico de vacinação (através da revisão dos dados de vacinação), estado de gravidez, resultados laboratoriais e medidas de controle. Esses dados são digitados no Sistema Nacional de Informações sobre Doenças de Notificação (SINAN) no nível municipal ou regional, transferidos ao nível estadual e finalmente ao nível nacional, ou seja, ao Ministério da Saúde.

Um caso suspeito de rubéola é definido como um indivíduo de qualquer idade com queixa de febre, exantema e linfadenopatia cervical, occipital ou pós-auricular; ou qualquer indivíduo cuja suspeita de rubéola seja levantada por um profissional da saúde. Os casos suspeitos são confirmados (1) por exames laboratoriais, caso os anticorpos IgM específicos anti-rubéola sejam detectados por um ensaio imunoenzimático (Behringwerke AG Diagnostica); (2) por vínculo epidemiológico, no caso de exposição a um caso de rubéola confirmado laboratorialmente entre 12 e 23 dias antes do início dos sintomas; ou (3) clinicamente, quando o caso suspeito apresentar febre, exantema e linfadenopatia, não forem realizados exames laboratoriais, ou quando o caso suspeito não puder ser descartado com base no vínculo epidemiológico com outras doenças exantemáticas (ex: dengue) ou pela associação temporal com a administração de vacina de rubéola.

A fim de descrever a epidemiologia da rubéola na Paraíba entre 1999 e 2005, analisamos dados do SINAN usando o Epi Info versão 6.04b. Calculamos a incidência anual de rubéola por 100.000 habitantes utilizando o número de casos confirmados de rubéola como numerador e as estimativas do censo populacional anual como denominador. O teste do quiquadrado foi usado para comparar as taxas de incidência entre os grupos etários e as proporções.

\section{Vigilância da SRC}

A vigilância da SRC foi iniciada em 2000 através (a) do acompanhamento de gestantes com rubéola e (b) notificação de lactentes com suspeita de SRC. Em todos os casos suspeitos de SRC, os prontuários médicos dos pacientes foram revisados e foram realizadas investigações ao nível hospitalar e domiciliar através do preenchimento de um questionário padronizado e da coleta de uma amostra sangüínea para a detecção de IgM e IgG específicos, caso esse teste não tenha 


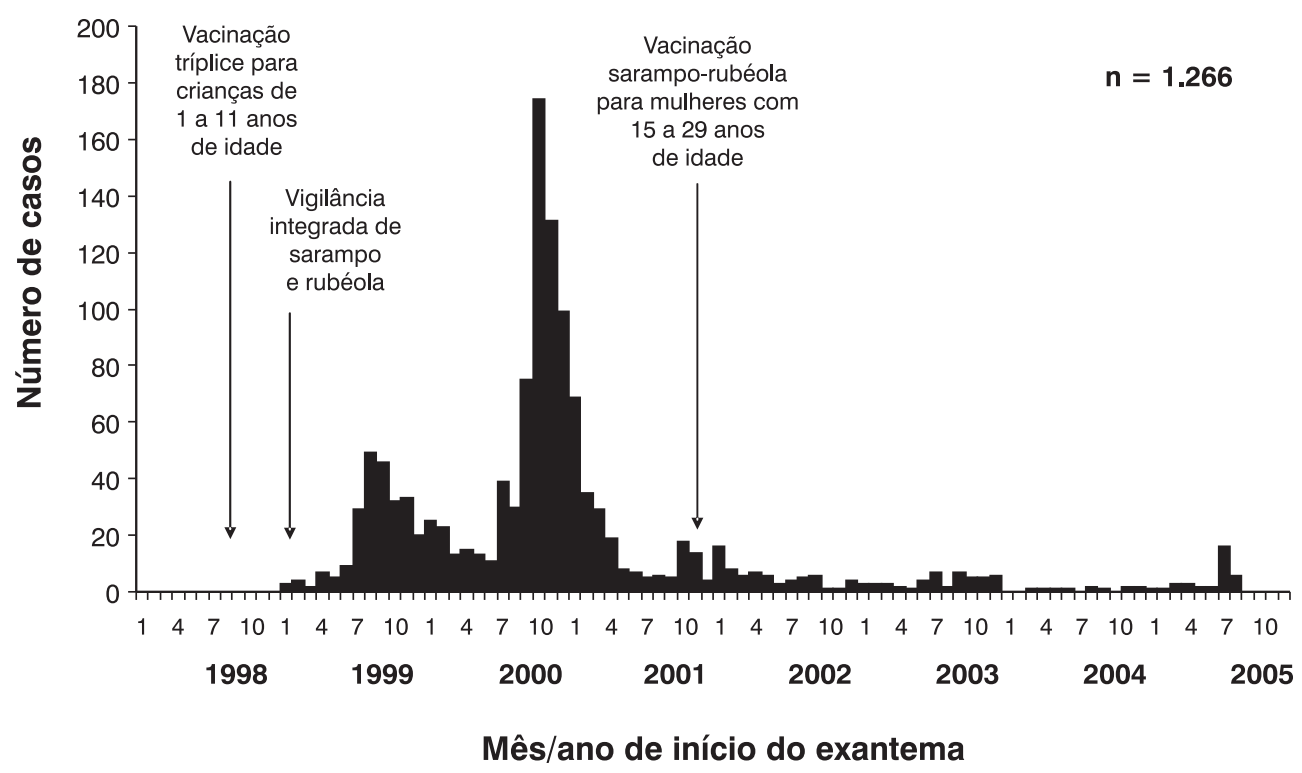

Figura 1 - Casos confirmados de rubéola de acordo com o mês de início das erupções, estado da Paraíba, Brasil, 1999-2005

sido realizado no momento do nascimento. Em 2001, uma vigilância sentinela ativa da SRC foi estabelecida em uma maternidade da capital do estado, João Pessoa, que serve de serviço de referência para o estado.

Um caso suspeito de SRC foi definido como 1) um lactente cuja mãe relatou ser caso confirmado de rubéola durante a gravidez; ou 2) um lactente com manifestações compatíveis com SRC. Os casos suspeitos de SRC foram classificados de acordo com as definições de caso padronizadas pela Organização Mundial da Saúde como caso confirmado laboratorialmente, compatível clinicamente ou infecção por rubéola congênita (IRC) ${ }^{8}$. Gestações com desfecho desconhecido ou lactentes cujas manifestações compatíveis com a SRC não foram avaliadas foram considerados como perda de seguimento.

As notificações de SRC foram digitadas no SINAN e analisadas usando-se o Epi Info versão 6.04b. A incidência da SRC foi calculada utilizando-se o número total de lactentes com diagnóstico compatível ou confirmado de SRC como numerador e as estimativas anuais do censo para a população com menos de 1 ano de idade como denominador.

\section{Resultados}

\section{Epidemiologia da rubéola}

Entre 1999 e 2005, 5.954 casos suspeitos de rubéola foram notificados na Paraíba; 1.391 (23\%) casos suspeitos não puderam ser classificados devido à insuficiência ou inconsistência dos dados clínicos, epidemiológicos e laboratoriais. Do restante, $1.266(21 \%)$ casos foram confirmados, 766 (61\%) por exames laboratoriais, 323 (25\%) por vínculo epidemiológico e 177 (14\%) por critério clínico. Dentre os 3.297 (55\%) casos descartados, 2.022 (61\%) apresentaram testes de IgM negativos para rubéola e 749 (23\%) apresentaram resultados negativos de IgM para sarampo. O intervalo mediano entre o início dos sintomas e a data da coleta da amostra foi de 5 dias nos casos confirmados laboratorialmente ( $n=630$; intervalo: 0-248 dias) e de 5 dias nos casos descartados ( $n=1.894$; intervalo: 0-278 dias). O diagnóstico não foi realizado em 1.259 (38\%) casos descartados e foi desconhecido em 672 (20\%); 903 (27\%) relataram doença exantemática não especificada, 425 (13\%) dengue, 22 (1\%) exantema súbito, $9(0.03 \%)$ reações adversas à vacina, 3 febre escarlatina, 2 eritema infeccioso e 2 infecção enteroviral.

Dos 1.266 casos confirmados, 239 (19\%) ocorreram em 1999,648 (51\%) em 2000, 219 (17\%) em 2001 e os restantes 160 (13\%) entre 2002 e 2005 . O número de casos confirmados teve um pico em outubro de $2000(n=174)$ (Figura 1$)$. A incidência de rubéola diminuiu de 18,8 por 100.000 em 2000 para 0,9 em 2005 (Tabela 1).

A mediana de idade entre os pacientes foi de 17,5 anos em 1999 (intervalo: 0-53) e diminuiu para 8 anos em 2002 (intervalo: 0-75) e para 2 anos em 2005 (intervalo:0-42). Do total de casos, 749 (59\%) dos pacientes eram do sexo feminino e 372 (29\%) eram mulheres com idades entre 15 e 29 anos; a proporção de mulheres com idades entre 15 e 29 anos entre os pacientes diminuiu de $32 \%$ entre 1999 e 2001 para $13 \%$ entre 2002 e 2005 ( $p<0,01$ ). Casos confirmados de rubéola foram notificados em 64 mulheres grávidas; destes, a confirmação se deu por exames laboratoriais em 50 (78\%), por vínculo epidemiológico em 12 (19\%) e por exame clínico em 2 (3\%). Todos os casos de pacientes grávidas com rubéola foram notificados entre 1999 e 2003 , dos quais 43 (67\%) apresentaram a doença em 2000. A mediana de idade das 
Tabela 1 - Distribuição dos casos confirmados de rubéola por idade, gênero e estado de gravidez, Paraíba, Brasil, 1999-2005

\begin{tabular}{|c|c|c|c|c|c|c|c|}
\hline \multirow[b]{2}{*}{ Variáveis } & \multicolumn{7}{|c|}{ Número de casos (\%) } \\
\hline & 1999 & 2000 & 2001 & 2002 & 2003 & 2004 & 2005 \\
\hline \multicolumn{8}{|l|}{ Faixa etária (anos) } \\
\hline$<1$ & $12(5)$ & $31(5)$ & $20(9)$ & $10(15)$ & $18(38)$ & $2(18)$ & $11(32)$ \\
\hline $1-13$ & $45(19)$ & $98(15)$ & $58(26)$ & $30(45)$ & $21(44)$ & $7(64)$ & $18(53)$ \\
\hline $14-19$ & $81(34)$ & $197(30)$ & $43(20)$ & $5(7)$ & $4(8)$ & $1(9)$ & $1(3)$ \\
\hline $20-29$ & $48(20)$ & $200(31)$ & $68(31)$ & $11(16)$ & $2(4)$ & $0(0)$ & $2(6)$ \\
\hline$>30$ & $32(13)$ & $103(16)$ & $30(14)$ & $11(16)$ & $3(6)$ & $1(9)$ & $2(6)$ \\
\hline Total* & 239 & 648 & 219 & 67 & 48 & 11 & 34 \\
\hline Sexo (feminino) & $136(57)$ & $392(61)$ & $129(59)$ & $45(67)$ & $22(46)$ & $4(36)$ & $23(68)$ \\
\hline Mulheres de 15 a 29 anos & $66(28)$ & $223(34)$ & $64(29)$ & $12(18)$ & $5(10)$ & $0(0)$ & $3(9)$ \\
\hline Mulheres grávidas & $4(2)$ & $43(7)$ & $11(5)$ & $5(8)$ & $1(2)$ & $0(0)$ & $0(0)$ \\
\hline
\end{tabular}

* 21 casos sem dados para idade em 1999 e 19 em 2000.

pacientes grávidas foi de 22 anos, variando de 14 a 40 anos $(n=63)$.

Em 2000, considerado como um ano de epidemia, 397 ( $61 \%$ ) casos confirmados de rubéola ocorreram entre adolescentes e adultos jovens com idades entre 14 e 29 anos, com uma relação masculino:feminino de 2:3. A maior incidência de rubéola ocorreu entre as crianças com menos de 1 ano de idade (47/100.000) seguida de indivíduos com idades entre 14 e 19 anos (42/100.000) e entre 20 e 29 anos (34/100.000) (Tabela 1). Com relação às crianças com idades entre 1 e 13 anos (10/100.000), o grupo etário-alvo durante a campanha de vacina tríplice em 1998, a incidência de rubéola aumentou 4,5 vezes entre as crianças com menos de 1 ano de idade (IC95\% 3,0-6,8), 4,0 vezes entre os indivíduos com idades entre 14 e 19 anos (IC95\% 3,2-5,1) e 3,3 vezes entre aqueles com idades entre 20 e 29 anos (IC 95\% 2,6-4,2).

\section{Epidemiologia da SRC}

Casos de rubéola foram confirmados em 64 mulheres grávidas entre 1999 e 2003. Destes, 24 (38\%) tiveram perda de seguimento; 12 (50\%) foram notificados entre 1999 e 2000 , $6(25 \%)$ em 2000, 5 (21\%) em 2002 e 1 (4\%) em 2003. Das $40(72 \%)$ mulheres grávidas restantes, $3(5 \%)$ apresentaram aborto espontâneo, 1 (2\%) natimorto e 36 (56\%) deram à luz lactentes vivos (Tabela 2). Dos 141 lactentes com suspeita de SRC notificados entre 1999 e 2005, seis apresentaram perda de seguimento. Um total de 171 casos suspeitos de SRC foram investigados, 167 (98\%) foram testados para IgM e 18 (11\%) apresentaram resultados positivos; e amostras sangüíneas foram coletadas de 154 (92\%) nos primeiros 6 meses de vida. Quatorze (8\%) lactentes foram classificados como casos confirmados laboratorialmente de SRC, dois (1\%) foram clinicamente compatíveis, quatro (2\%) apresentaram IRC e 151 (88\%) foram descartados. Dos casos descartados, $133(88 \%)$ apresentaram um resultado negativo para a pesquisa de anticorpos IgM contra a rubéola em uma amostra coletada durante os 6 primeiros meses de vida.
A incidência de SRC por 1.000 crianças com menos de 1 ano de idade foi de 0,01 em $1999(n=1), 0,03$ em 2000 ( $n=$ 2) e de 0,20 em 2001 ( $n=13)$. Entre 2002 e 2005, nenhum caso de SRC compatível ou classificado como IRC foi confirmado. Os casos de SRC confirmados ou compatíveis nascidos em 2001 ocorreram em oito (4\%) dos 224 municípios, com incidência de SRC entre 1 a 16 casos por 1.000 crianças com menos de 1 ano de idade.

Dos 16 lactentes com SRC nascidos entre 1999 e 2001, nove ( $56 \%$ ) apresentaram cardiopatia congênita, oito (50\%) apresentaram comprometimento auditivo, como manifestação única da SRC em cinco (31\%), e seis (38\%) apresentaram catarata. Um (6\%) lactente apresentou essas três manifestações e cinco (31\%) apresentaram duas dessas manifestações. Seis (38\%) lactentes tiveram púrpura, quatro $(25 \%)$ apresentaram icterícia, dois (13\%) tiveram hepatoesplenomegalia e um (6\%) apresentou retardamento mental. Três (19\%) lactentes nasceram antes de 37 semanas de gestação e 11 (69\%) apresentaram peso ao nascer menor que 2.500 g. Cinco (31\%) lactentes com confirmação de SRC apresentaram desfecho fatal até o terceiro mês de vida, quatro $(80 \%)$ no primeiro mês. Todos tiveram peso ao nascer menor que $2.500 \mathrm{~g}$, cardiopatia congênita e púrpura.

Dos 63 casos suspeitos de SRC com rubéola materna durante a gravidez, 14 foram confirmados laboratorialmente, um apresentou SRC clinicamente compatível, quatro apresentaram IRC, 42 foram descartados e dois tiveram perda de seguimento. Dos 22 casos suspeitos de SRC com rubéola materna durante o primeiro trimestre de gestação, 12 (55\%) foram confirmados laboratorialmente, três (14\%) apresentaram IRC e sete foram descartados. A mediana de idade das mães que tiveram bebês em que a SRC foi confirmada foi de 26 anos, variando de 23 a 27 anos $(n=5)$; nenhuma havia recebido a vacina anteriormente.

\section{Discussão}

Neste estudo, documentamos a epidemiologia da rubéola e da SRC no estado da Paraíba, Brasil, entre 2000 e 2005. A 
Tabela 2 - Desfecho da gravidez entre mulheres com confirmação de rubéola e classificação de lactentes com suspeita de síndrome de rubéola congênita, estado da Paraíba, Brasil, 1999-2005

\begin{tabular}{|c|c|c|c|}
\hline \multirow[b]{2}{*}{ Número de indivíduos } & \multicolumn{2}{|c|}{ Tipo de entrada no sistema de vigilância da SRC8* } & \multirow[b]{2}{*}{ Total } \\
\hline & $\begin{array}{l}\text { Seguimento de mulheres } \\
\text { grávidas com } \\
\text { confirmação de rubéola }\end{array}$ & $\begin{array}{c}\text { Lactentes com suspeita } \\
\text { de SRC identificados na } \\
\text { maternidade }\end{array}$ & \\
\hline Coorte inicial & 64 & 141 & 205 \\
\hline Perda de seguimento & 24 & 6 & 30 \\
\hline Aborto & 3 & - & 3 \\
\hline Natimorto & 1 & - & 1 \\
\hline \multicolumn{4}{|l|}{ Lactentes } \\
\hline Investigados & 36 & 135 & 171 \\
\hline Com teste de IgM & 33 & 134 & 167 \\
\hline IgM positivos & 9 & 9 & 18 \\
\hline SRC confirmada por exames laboratoriais & 5 & 9 & 14 \\
\hline SRC clinicamente compatível & 1 & 1 & 2 \\
\hline $\operatorname{IRC}^{+}$ & 4 & 0 & 4 \\
\hline Descartados & 26 & 125 & 151 \\
\hline
\end{tabular}

\footnotetext{
* Síndrome de rubéola congênita
}

+ Infecção por rubéola congênita.

vacinação contra a rubéola direcionada às crianças com idades entre 1 e 11 anos em 1998 não foi suficiente para prevenir um surto entre os adultos jovens suscetíveis em 2000, com subseqüente alta incidência de SRC em 2001. Todavia, o surto parece não ter sido somente devido à cobertura vacinal subótima, mas também devido à alta intensidade de transmissão e um considerável grupo suscetível na população de adultos jovens não incluídos da população-alvo da vacinação.

A vigilância ativa prospectiva nas maternidades e um seguimento completo de pacientes grávidas com rubéola foram essenciais para a documentação da carga de SRC em 2001 e o impacto das estratégias de vacinação para reduzir essa carga nos anos subseqüentes. Apesar da vigilância intensa, a verdadeira carga de SRC é provavelmente subestimada por várias razões: 1) falta de pacientes que procuram atendimento médico devido à manifestação clínica convencional da rubéola como doença exantemática leve; 2) subnotificação do estado de gravidez nos relatos de casos de rubéola, 3) perda de seguimento de um grande número de pacientes grávidas com rubéola e de lactentes com suspeita de SRC, 4) falta de informações sobre sinais e sintomas nos prontuários médicos de pacientes com suspeita de SRC, 5) ausência de avaliação do comprometimento auditivo de todos os casos com suspeita de SRC, e 6) falta de seguimento de casos de IRC que venham a manifestar, posteriormente, seqüelas relacionadas à SRC.

A proporção de casos de SRC diagnosticados com cardiopatia foi comparável àquela observada em outros estudos com seguimento completo, nos quais aproximadamente $40-50 \%$ das crianças com SRC apresentaram cardiopatia ${ }^{9}$. Apenas metade dos nossos casos de SRC apresentou comprometimento auditivo, embora em algumas séries até 60 a $90 \%$ dos pacientes com SRC sejam afetados ${ }^{9-11}$. Um terço dos lactentes com SRC apresentaram um desfecho fatal nos primeiros 3 meses de vida e uma alta proporção de casos suspeitos de SRC foi oportunamente testada para anticorpos IgM contra a rubéola. Contudo, é possível que casos com desfecho fatal precoce ou que apresentaram poucos sinais indicativos de SRC não tenham sido computados e não tenham sido avaliados quanto ao comprometimento auditivo precoce.

A incidência anual da SRC foi estimada em 0,2 por 1.000 crianças com menos de 1 ano de idade no estado da Paraíba durante o ano de 2001, mais baixa que a incidência relatada em outros estudos tanto no Brasil como em outros países após os surtos de rubéola em populações não-vacinadas ${ }^{3,12,13}$. No Brasil, foram documentadas incidências de 0,6 e 0,9 por 1.000 nascidos vivos em estudos prospectivos e retrospectivos após os surtos de rubéola entre 1999 e $2001^{3,12}$. Em outros países em desenvolvimento, as taxas de SRC por 1.000 nascidos vivos após surtos de rubéola variaram de 0,4 na Jamaica durante o ano de 1995 a 2,2 no Panamá entre 1986 e $1987^{13}$. No entanto, essa incidência estadual anual na Paraíba mascarou uma incidência 2,5 vezes maior ( 0,5 por 1.000 crianças com menos de 1 ano) entre maio e agosto de 2001, 7 a 10 meses após o mês de pico da rubéola e em certos municípios em que a incidência foi 5 a 80 vezes maior que a incidência estadual. A falta de seguimento de $38 \%$ das mulheres grávidas com confirmação de rubéola entre 1999 e 2003 também pode ter limitado nossas estimativas da incidência de SRC, embora as tendências teriam sido semelhantes se todas essas 
mulheres tivessem filhos com SRC, já que a maioria delas foram notificadas no ano 2000.

Observamos que durante o ano da epidemia, a incidência da rubéola foi maior entre as crianças com menos de 1 ano, adolescentes e adultos jovens; a mediana de idade à infecção foi de 19 anos em 2000. Antes da implementação da vacinação contra a rubéola, a rubéola era uma doença infantil comum no Brasil, com uma média de idade de infecção de 6 anos e com epidemias acontecendo aproximadamente a cada 6 a 9 anos em áreas urbanas ${ }^{14,15}$. Infelizmente, dados de vigilância da rubéola no estado da Paraíba não estavam disponíveis para comparação nos anos anteriores a 1999 para afirmar que houve uma mudança na distribuição etária dos casos resultantes da campanha de vacinação de 1998. Uma mudança na média de idade da primeira infecção é um efeito indireto importante de imunização em massa, resultando em períodos mais longos entre as epidemias. Tal período de baixa incidência durante a mudança da distribuição etária de prévacinação para pós-vacinação dos indivíduos suscetíveis, chamado de "período de lua-de-mel", pode ser seguido de um novo aumento no número de $\operatorname{casos}^{16}$. A transmissão endêmica-epidêmica contínua entre adultos foi observada após a implementação da vacinação infantil de rotina em vários países, até mesmo naqueles com níveis de cobertura relativamente altos, devido principalmente a um aumento no risco de infecção por rubéola entre jovens adultos suscetíveis ${ }^{17-22}$. Para prevenir a SRC, muitos países adotaram a vacinação contra a rubéola entre estudantes e mulheres em idade reprodutiva após ou conjuntamente com a implementação do esquema vacinal infantil.

Este estudo fornece dados populacionais que documentam a incidência da rubéola e da SRC no estado da Paraíba, Brasil, entre 2000 e 2005. A incidência maior de rubéola entre adultos entre os anos de 1999 e 2000 e o aumento na detecção de casos de SRC, tanto na Paraíba como no resto do Brasil, levou a esforços intensos de vacinação de mulheres em idade reprodutiva. Embora seja esperada uma baixa incidência de rubéola nos anos seguintes aos surtos, a redução considerável na incidência da rubéola e da SRC observada após 2001, a despeito da intensificação da vigilância para se detectar casos suspeitos, reflete um valor preditivo positivo baixo da vigilância de ambas as doenças e demonstra a eficácia das estratégias de vacinação implementadas. A campanha de vacinação entre mulheres em idade reprodutiva em 2001 após o surto aparentemente reduziu o número de indivíduos suscetíveis remanescentes nesse grupo. Contudo, jovens adultos do sexo masculino, que não foram incluídos no esquema de vacinação, podem exercer um papel importante na transmissão de rubéola no futuro. Entretanto, à medida que coortes sucessivas de crianças (do sexo masculino e feminino) atingem a idade adulta, o risco de transmissão da rubéola é reduzido.

Além disso, alguns municípios não atingiram a cobertura vacinal de $95 \%$, conforme recomendado, nas campanhas anteriores ou na vacinação infantil de rotina, aumentando o risco de surtos no futuro. A alta cobertura vacinal de rotina contra a rubéola ao nível estadual, municipal e local juntamente com um sistema de vigilância de alta qualidade é importante para a eliminação da doença no Brasil até 2010. Nas populações com cobertura vacinal subótima, e mesmo nas populações parcialmente vacinadas, quando a vacina encontra-se disponível apenas nos setores privados, podem ocorrer aumentos futuros na incidência da $\mathrm{SRC}^{23}$. Felizmente no Brasil, a vacina é oferecida através do serviço de saúde pública. No entanto, os níveis de cobertura ou proteção abaixo do mínimo recomendado resultaram nos surtos de rubéola, como no caso do estado da Paraíba. Portanto, recomendamos monitorar a cobertura de rotina contra a rubéola a fim de identificar as áreas e subgrupos com maior risco, intensificar a vacinação em áreas com baixa cobertura além de realizar análises dos dados de vigilância da rubéola a fim de identificar e investigar aglomerados de casos suspeitos de rubéola, com a adoção oportuna de medidas de controle para prevenir a SRC. Finalmente, para documentar o impacto das estratégias de vacinação contra a rubéola, os esforços devem se concentrar em manter a alta sensibilidade da vigilância da rubéola e da SRC em períodos de baixa incidência de ambas as doenças.

\section{Agradecimentos}

Agradecemos à Dra. Shamya Lewis Saad Rached, neonatologista do Instituto Cândida Vargas, pelo empenho no estabelecimento da vigilância sentinela da SRC e dedicação à detecção e notificação dos casos suspeitos de SRC; ao pessoal do Laboratório Central de Saúde Pública do estado da Paraíba, pelo manejo das amostras sangüíneas e pesquisas de anticorpos; a todos os membros dos serviços municipais e estaduais de vigilância e imunização do estado da Paraíba, em especial aqueles de João Pessoa, pelos seus esforços intensos em relação à vigilância da doença e à vacinação contra a rubéola; e a Henry Walke e Douglas L. Hatch pela revisão cuidadosa do artigo.

\section{Referências}

1. Centers for Disease Control and Prevention (CDC). Rubella prevention. MMWR Morb Mortal Wkly Rep. 1984;33:301-10; 315-8.

2. Cutts FT, Robertson SE, Diaz-Ortega JL, Samuel R. Control of rubella and congenital rubella syndrome (CRS) in developing countries, Part 1: Burden of disease from CRS. Bull World Health Organ. 1997;75: 55-68.

3. Lanzieri TM, Segatto TC, Siqueira MM, de Oliveira Santos EC, Jin L, Prevots DR. Burden of congenital rubella syndrome after a community-wide rubella outbreak, Rio Branco, Acre, Brazil, 2000 to 2001. Pediatr Infect Dis J. 2003;22: 323-9.

4. Lanzieri TM, Prevots DR, Dourado I.Surveillance of congenital rubella syndrome in Brazil, 1995-2005. J Pediatr Infect Dis. 2007;2:15-22. 
5. Prevots DR, Parise MS, Segatto TC, Siqueira MM, dos Santos ED, Ganter B, et al. Interruption of measles transmission in Brazil, 2000-2001. J Infect Dis. 2003;187 Suppl 1:S111-20.

6. Accelerated control of rubella and prevention of congenital rubella syndrome, Brazil. Wkly Epidemiol Rec. 2002;77:169-75.

7. Pan American Health Organization (PAHO). 44th Directing Council. Resolution CD44.R1: sustaining immunization programs - elimination of rubella and congenital rubella syndrome (CRS). 2003.http://www.paho.org/English/GOV/CD/cd44-fr-e.pdf. Acesso: 17/03/2007.

8. World Health Organization. Report of a meeting on preventing congenital rubella syndrome: immunization strategies, surveillance needs. Geneva: WHO; 2000.

9. Reef SE, Plotkin S, Cordero JF, Katz M, Cooper L, Schwartz B, et al. Preparing for elimination of congenital Rubella syndrome (CRS): summary of a workshop on CRS elimination in the United States. Clin Infect Dis. 2000;31: 85-95.

10. Givens KT, Lee DA, Jones T, Ilstrup DM. Congenital rubella syndrome: ophthalmic manifestations and associated systemic disorders. Br J Ophthalmol. 1993;77: 358-63.

11. Peckham C. Congenital rubella in the United Kingdom before 1970: the prevaccine era. Rev Infect Dis. $1985 ; 7$ Suppl 1:S 11-6.

12. Lanzieri TM, Parise MS, Siqueira MM, Fortaleza BM, Segatto TC, Prevots DR. Incidence, clinical features and estimated costs of congenital rubella syndrome after a large rubella outbreak in Recife, Brazil, 1999-2000. Pediatr Infect Dis J. 2004;23: 111622.

13. Robertson SE, Featherstone DA, Gacic-Dobo M, Hersh BS. Rubella and congenital rubella syndrome global update. Rev Pan Am Salud Publica. 2003;14: 306-15.

14. Schatzmayr HG. Aspects of rubella infection in Brazil. Rev Infect Dis. 1985;7 Suppl 1:S 53-5.
15. De Azevedo Neto RS, Silveira AS, Nokes DJ, Yang HM, Passos SD, Cardoso MR, et al.Rubella seroepidemiology in a nonimmunized population of Sao Paulo State, Brazil. Epidemiol Infect. 1994;113:161-73.

16. Halloran ME, Haber M, Longini IM, Struchiner CJ. Direct and indirect effects in vaccine efficacy and effectiveness. Am J Epidemiol. 1991;133: 323-31.

17. Accelerated control of rubella and prevention of congenital rubella syndrome, Brazil. Wkly Epidemiol Rec. 2002;77:169-75.

18. Bart KJ, Orenstein WA, Preblud SR, Hinman AR, Lewis FL, Williams NM. Elimination of rubella and congenital rubella from the United States. Pediatr Infect Dis. 1985;4: 14-21.

19. Centers for Disease Control and Prevention (CDC). Nationwide campaign for vaccination of adults against rubella and measles - Costa Rica, 2001. MMWR Morb Mortal Wkly Rep. 2001; 50:976-9.

20. Chile, Ministry of Health, Division de salud de las personas, Departamento de epidemiologia. La campana de vacunación contra la rubéola en Chile. Santiago: Ministry of Health; 2000.

21. Irons B, Lewis MJ, Dahl-Regis M, Castillo-Solórzano C, Carrasco PA, de Quadros CA. Strategies to eradicate rubella in the English speaking Caribbean. Am J Public Health. 2000;90:1545-9.

22. Pan American Health Organization (PAHO). Rubella vaccination of women of childbearing age in the Americas. EPI Newsletter. 2001;23:6.

23. Vynnycky E, Gay NJ, Cutts FT. The predicted impact of private sector MMR vaccination on the burden of Congenital Rubella Syndrome. Vaccine. 2003;21: 2708-19.

Correspondência:

Tatiana M. Lanzieri

Ministério da Saúde, Secretaria de Vigilância em Saúde, SCS,

Quadra 4, Bloco A, Edifício Principal, $6^{\circ}$ andar

CEP 70304-000 - Brasília, DF

Tel.: (61) 3212.8288 